\title{
High pressure Ca-VI phase between 158-180 GPa: Stability, electronic structure and superconductivity
}

\author{
M. Aftabuzzaman, A. K. M. A. Islam* \\ Department of Physics, Rajshahi University, Rajshahi-6205, Bangladesh
}

\begin{abstract}
We have performed $a b$ initio calculations for new high-pressure phase of Ca-VI between 158-180 GPa. The study includes elastic parameters of mono- and polycrystalline aggregates, electronic band structure, lattice dynamics and superconductivity. The calculations show that the orthorhombic Pnma structure is mechanically and dynamically stable in the pressure range studied. The structure is superconducting in the entire pressure range and the calculated $T_{\mathrm{c}}(\sim 25 \mathrm{~K})$ is maximum at $\sim 172 \mathrm{GPa}$, where the transfer of charges from $4 s$ to $3 d$ may be thought to be completed.
\end{abstract}

PACS number(s): 74.70.Ad, 62.20.de, 71.20.-b, 74.20.Pq, 74.25.Kc, 74.62.Fj

Keywords: Calcium; High pressure; Electronic band structure; Phonon spectrum; Elastic constants; Superconductor.

\section{Introduction}

The physical properties of some of the alkaline-earth metals like $\mathrm{Ca}, \mathrm{Sr}$, and $\mathrm{Ba}$ have been studied both theoretically and experimentally in view of $s-d$ electron transfer under high pressure [1]. Calcium experiences a series of phase transitions under pressure. It is a facecentered cubic (fcc) (Ca-I) lattice at ambient conditions. Under compression the structural transitions are [2]: body-centered cubic (Ca-II) $\rightarrow$ simple cubic (Ca-III) $\rightarrow P 4_{1} 2_{1} 2 \quad(\mathrm{Ca}-$ IV) $\rightarrow C m c a(C a-V)$. Like $\mathrm{Ba}$ [3-6] and $\mathrm{Sr}$ [4-7], Ca was found to show superconductivity under pressure. Dunn and Bundy [8] first observed the superconductivity of $\mathrm{Ca}$ at $44 \mathrm{GPa}$ as a small drop in the electrical resistance at $2 \mathrm{~K}$ within the sc structure. Subsequently it was demonstrated by Okada et al. [9] that the superconducting transition temperature $T_{\mathrm{c}} \leq 3 \mathrm{~K}$ above $85 \mathrm{GPa}$, which increases linearly with pressure up to $15 \mathrm{~K}$ at $150 \mathrm{GPa}$. Furthermore $T_{\mathrm{c}}$ is experimentally reported by Yabuuchi et al. [10] to rise with increase in pressure in Ca-IV $\left(P_{1}{ }_{2} 2\right)$ and $\mathrm{Ca}-\mathrm{V}(\mathrm{Cmca})$ phases, which reaches to $25 \mathrm{~K}$ at $161 \mathrm{GPa}$.

A number of theoretical works on the high-pressure polymorphs of $\mathrm{Ca}$ have been carried out recently [11-14]. Yao et al. [13] have previously made theoretical calculation and reported a Pnma structure between 110-130 GPa which they identified as Ca-IV. Recently Ishikawa et al. [14] reinvestigated the phase diagram of calcium at high pressure, taking all theoretically predicted and experimentally reported structures into account. They proposed two phases CaVI (Pnma, 117-135 GPa) and Ca-VII (I4/mcm $(00 \gamma)$ above $135 \mathrm{GPa}$. Additionally they found the Pnma phase after Ca-V ( $\mathrm{Cmca})$, which they introduced from the data of Nakamoto et al. [2]. This structure is same as proposed by Yao et al. [13] Ishikawa et al. [14] also remarked that $\mathrm{Ca}-\mathrm{V}(109-117 \mathrm{GPa})$ may be realized in the narrow pressure region of less than $10 \mathrm{GPa}$ and the highest superconducting $T_{\mathrm{c}}$ of $25 \mathrm{~K}$ experimentally reported may be realized in the $\mathrm{Ca}-\mathrm{VI}$ or Ca-VII phase.

\footnotetext{
${ }^{*}$ Corresponding author: azi46@ru.ac.bd
} 
Lei et al. [11] carried out a theoretical study on the superconductivity of Ca within the rigid muffin-tin approximation (RMTA) and utilized the approximate formula $\left\langle\omega^{2}\right\rangle=0.5 \theta_{\mathrm{D}}{ }^{2}$ without considering the distribution of the phonon density of states. This approximation yields a much larger $T_{\mathrm{c}}$ compared to experiment. Gao et al. [15] have presented a study on the electronic behaviour, lattice dynamics and superconductivity of sc Ca up to $100 \mathrm{GPa}$. Several interesting phenomena are observed. Firstly, it is suggested that the electronic topology transitions (ETT) at the X point is responsible for the observed anomaly in electrical resistance at $40 \mathrm{GPa}$. Secondly, they predict that the sc $\mathrm{Ca}$ is dynamically unstable in the pressure range over its existence. This observation either suggests a need for the reexamination of the sc structure, or indicates a large anharmonic effect which stands to stabilize the phase. Lastly the observed increase of $T_{\mathrm{c}}$ under pressure is mainly due to the enhanced electron-phonon coupling (EPC) matrix element and the larger electronic DOS. The latter results from the transfer of charges from $4 s$ to $3 d$ under pressure.

Very recently Nakamoto et al. [2] using synchrotron radiation carried out x-ray diffraction measurements of calcium at pressure up to $172 \mathrm{GPa}$ at room temperature and noted a highpressure phase "Ca-VI" above $158 \mathrm{GPa}$. An orthorhombic Pnma structure was determined by a Rietveld analysis. The obtained structure matched to the ones predicted by Yao et al. [13] well. However, their predicted pressure range did not match the experimentally observed pressure range. Nakamoto et al. [10] in a previous paper reported that the highest $T_{\mathrm{c}}$ of $25 \mathrm{~K}$ in the element was observed at $161 \mathrm{GPa}$ but the phase was previously considered to be $\mathrm{Ca}-\mathrm{V}$ $(\mathrm{Cmca})$. But the latest x-ray measurement indicates that the phase could possibly be a mixture of $\mathrm{Ca}-\mathrm{V}$ and $\mathrm{Ca}-\mathrm{VI}$, with clear $\mathrm{Ca}-\mathrm{VI}$ at $172 \mathrm{GPa}$. They suggested the necessity of phase study at low temperature and high pressure. Yin et al. [16], on the other hand, theoretically showed that the Pnma structure is favored at pressure over $140 \mathrm{GPa}$. Previously Ahuja et al. [17] calculated the top and bottom of the Ca $3 p, 3 d$ and $4 s$ bands as a function of pressure and indicated that the $s-d$ electronic transfer completes at $V / V_{0} \sim 0.2$, where the $4 s$ band becomes empty. However, the structural sequence used in the calculation $\mathrm{fcc} \rightarrow \mathrm{bcc} \rightarrow \mathrm{sc}$ $\rightarrow$ hcp is different from that found in the experiments $[2,10]$. Therefore the $s-d$ transfer cannot be considered complete even in the Ca-IV phase and hence it seems that $T_{\mathrm{c}}$ may increase above $25 \mathrm{~K}$ on further compression above $161 \mathrm{GPa}$. Higher-pressure experiments are now in progress by Nakamoto et al. [2].

The structural phase transitions reported so far and the interesting superconductivity in CaVI (Pnma) have brought new avenues in front of us. The present work will therefore focus on the newly discovered phase "Ca-VI" (Pnma) at pressure above $158 \mathrm{GPa}$ to shed further light on the results reported earlier, and particularly new results between 172-180 GPa. An analysis of the crystal structure, electronic band structures, lattice dynamics and EPC of the Ca-VI wi be made. The dynamic stability would also be an issue at hand. The calculation of elastic parameters of mono- and poly-crystalline $\mathrm{Ca}-\mathrm{VI}$ in the pressure region above $158 \mathrm{GPa}$ would enable us to comment on some elastic behavior as well as the mechanical stability of Ca-VI.

\section{Method of computations}

The ab-initio calculations were performed using DFT formalism as implemented in the CASTEP [18]. The geometrical optimization was done for high-pressure orthorhombic phase of $\mathrm{Ca}$ (space group $62 \mathrm{Pnma}$ ) treating the system as metallic with density mixing treatment of electrons. The generalized gradient approximation (GGA) of Perdew, Burke, and Ernzerhof for solids [19] potential has been incorporated for the simulation. We have used a $6 \times 8 \times 9$ Monkhorst grid to sample the Brillouin zone. All structures have been fully optimized until internal stress and forces on each atom are negligible. For all relevant calculations the plane wave basis set cut-off used is $310 \mathrm{eV}$ and the convergence criterion is $0.5 \times 10^{-5} \mathrm{eV} /$ atom.

Calculations of phonon spectra, electron-phonon (e-ph) coupling and phonon density of states were performed using plane waves and pseudopotentials with QUANTUM ESPRESSO [20]. We employed ultrasoft Vanderbilt pseudopotentials [21], with a cut-off of 50 Ryd for the wave functions, and $400 \mathrm{Ryd}$ for the charge densities. The $k$-space integration for the electrons was approximated by a summation over a $8 \times 8 \times 8$ uniform grid in reciprocal space, 
with a Gaussian smearing of 0.02 Ryd for self-consistent cycles. Dynamical matrices and e-ph linewidths were calculated on a uniform $2 \times 2 \times 2$ grid in phonon $q$-space. Phonon dispersions and DOS were then obtained by Fourier interpolation of the dynamical matrices, and the Eliashberg function by summing over individual linewidths and phonons.

\section{Results and discussions}

\subsection{Geometrical optimization}

The optimized structure of the calcium orthorhombic phase VI (Pnma) is shown in figure 1. The lattice parameters and the converged atomic positions after the optimization are given in Table I. The nearest-neighbor distances for Ca-VI at pressures 158-180 GPa have been investigated. At $172 \mathrm{GPa}$, there are fourfold $d_{1}=2.2278 \AA$, twofold $d_{2}=2.2517 \AA$, and twofold $d_{3}=2.2529 \AA$, compared to corresponding values $2.268,2.273$ and $2.275 \AA$, respectively found experimentally by Nakamoto [2]. The next-nearest distance $d_{4}=2.7554 \AA$ is located much further than $d_{3}$. The coordination number for $\mathrm{Ca}-\mathrm{VI}$ is thus found to be 8 .

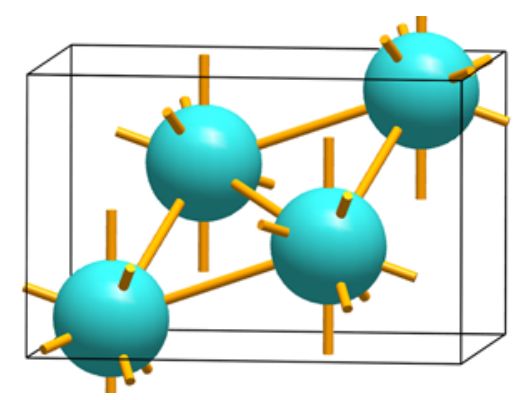

Figure 1. (Colour online) Unit cell of Ca-VI (Pnma) at $172 \mathrm{GPa}$.

Table 1. The optimized lattice parameters and fractional coordinates of Ca- VI (Pnma).

\begin{tabular}{cccccccc}
\hline $\begin{array}{c}\text { Pressure } \\
(\mathrm{GPa})\end{array}$ & $a(\AA)$ & $b(\AA)$ & $c(\AA)$ & $V\left(\AA^{3}\right)$ & \multicolumn{3}{c}{ Fractional coordinates } \\
\hline 158 & 4.2824 & 3.2390 & 2.7982 & 38.81 & 0.169654 & 0.250000 & 0.116566 \\
161 & 4.2764 & 3.2339 & 2.7892 & 38.57 & 0.169600 & 0.250000 & 0.116680 \\
165 & 4.2690 & 3.2280 & 2.7768 & 38.27 & 0.169491 & 0.250000 & 0.116721 \\
172 & 4.2555 & 3.2198 & 2.7554 & 37.75 & 0.169183 & 0.250000 & 0.116283 \\
$172^{\mathrm{a}}$ & 4.2760 & 3.2290 & 2.8810 & 39.78 & 0.170400 & 0.250000 & 0.114800 \\
180 & 4.2416 & 3.2124 & 2.7305 & 37.20 & 0.168789 & 0.250000 & 0.116106 \\
\hline
\end{tabular}

${ }^{\mathrm{a}} \operatorname{Expt}[2]$

\subsection{Elastic parameters of mono- and poly-crystalline Ca-VI and mechanical stability}

We performed systematic first-principles calculations of the elastic parameters of $\mathrm{Ca}-\mathrm{VI}$ single crystal, such as the elastic constants $C_{\mathrm{ij}}$, the bulk moduli $B$ and the shear moduli $G$ as a function of pressure (Table 2). These parameters are widely used for describing the elastic behavior of materials. The calculated nine independent elastic constants $\left(C_{11}, C_{12}, C_{13}, C_{23}\right.$, 
$\mathrm{C}_{22}, C_{33}, C_{44}, \mathrm{C}_{55}$ and $\left.C_{66}\right)$ of $\mathrm{Ca}-\mathrm{VI}$ at different pressures are found to satisfy the stability criteria for orthorhombic crystal [22]: $C_{\mathrm{ii}}>0(i=1,2 \ldots 6), C_{11}+C_{22}-2 C_{12}>0, C_{11}+C_{33}-2 C_{13}$ $>0, C_{22}+C_{33}-2 C_{23}>0$ and $\left(C_{11}+\mathrm{C}_{22}+C_{33}+2\left(C_{12}+C_{13}+C_{23}\right)>0\right.$.

Table 2. Elastic parameters (all in GPa, except for $v$ ) for mono- and poly-crystalline Ca-VI phase.

\begin{tabular}{ccccccccccccccc}
\hline \multirow{8}{*}{$\begin{array}{c}\text { Monocrystalline } \\
\text { (GPa) }\end{array}$} & $C_{11}$ & $C_{12}$ & $C_{13}$ & $C_{23}$ & $C_{22}$ & $C_{33}$ & $C_{44}$ & $C_{55}$ & $C_{66}$ & $B$ & $G$ & $Y$ & $v$ \\
\hline 158 & 928 & 315 & 356 & 469 & 626 & 508 & 267 & 165 & 187 & 475 & 153 & 415 & 0.355 \\
161 & 953 & 325 & 366 & 485 & 652 & 530 & 282 & 176 & 195 & 492 & 162 & 438 & 0.352 \\
165 & 976 & 338 & 375 & 497 & 664 & 546 & 287 & 182 & 200 & 505 & 166 & 449 & 0.352 \\
172 & 1013 & 360 & 388 & 534 & 713 & 577 & 307 & 185 & 209 & 534 & 173 & 468 & 0.354 \\
180 & 1064 & 395 & 406 & 583 & 782 & 580 & 325 & 200 & 230 & 561 & 174 & 473 & 0.360 \\
\hline
\end{tabular}

In order to study elastic anisotropy of Ca-VI we now calculate the Zener's anisotropy index $A$ [23]. For isotropic case $A=1$, while the deviation from unity measures the degree of elastic anisotropy. The calculated values of $A$ are $0.87,0.90,94$, and 0.97 at pressures of 158 , 161,172 , and $180 \mathrm{GPa}$, respectively. It is thus seen that Ca-VI approaches towards isotropy as pressure increases.

The crystals are usually synthesized in the form of polycrystalline substances. Thus the numerical estimates of the mechanical characteristics in the polycrystalline aggregates are desirable. The Voigt-Reuss-Hill procedure [24-26] is frequently used for estimating the elastic characteristics of polycrystalline materials using $C_{\mathrm{ij}}$-values for single crystals. Hill ${ }^{25}$ proved that the Viogt and Reuss equations represent upper and lower limits of true polycrystalline constants. He showed that the polycrystalline moduli $\left(B_{\mathrm{H}}, G_{\mathrm{H}}\right)$ are the arithmetic mean values of the moduli (monocrystalline values) in the Voigt $\left(B_{\mathrm{V}}, G_{\mathrm{V}}\right)$ and Reuss $\left(B_{\mathrm{R}}, G_{\mathrm{R}}\right)$ approximation. The Young's modulus $Y$ and Poisson's ratio $v$ are then computed from these values using the following relationship: $Y=9 B G /(3 B+G), v=(3 B-Y) / 6 B$. The bulk moduli $\left(B_{\mathrm{H}} \equiv B\right)$, shear moduli $\left(G_{\mathrm{H}} \equiv G\right)$, Young's moduli $(Y)$, and Poisson's ratios $(v)$ in the VoigtReuss-Hill approximation for polycrystalline $\mathrm{Ca}-\mathrm{VI}$ are presented in Table II. The expression for Reuss and Voigt moduli can be found elsewhere [27]. The calculated moduli reveal Ca-VI to be extremely hard material at the pressures under consideration.

According to Pugh [28], a given material is classified as brittle if $B / G<1.75$. The results of our calculations give $B / G \sim 3.03-3.10$, which should behave as a ductile material. The Poisson's ratios of covalent systems are known to be small $(v \sim 0.1)$, while those for ductile metallic materials $v$ are typically $\sim 0.33$ [29]. We can therefore assume that Ca-VI examined belong to metallic like system at all pressures.

The Debye temperatures using the formula and method given elsewhere [27] are found to be $742,762,771,785$, and $786 \mathrm{~K}$ at $158,161,165,172$, and $180 \mathrm{GPa}$, respectively. Unfortunately, there are no data, whether theoretical or experimental, available for comparison at the moment.

\subsection{Electronic band structure and DOS}

The calculated band structure and projected densities of states (DOS) to $s, p$, and $d$ orbitals of the Pnma structure at $172 \mathrm{GPa}$ are shown in figures 2 (a,b). Similar features of band structure and DOS are observed at other pressures considered here (figures not shown). In figure 2 (b) we plot the total and projected DOS for Ca in Ca-VI at $172 \mathrm{GPa}$. $E_{\mathrm{F}}$ falls slightly away from the pseudogap and into the all important $\mathrm{Ca} d$ bands, which gives the value of total 
DOS 1.74 states/eV. The Ca $d$ contribution changes from $\sim 95 \%$ to $\sim 96 \%$ of total DOS for a pressure change from 158 to $180 \mathrm{GPa}$.
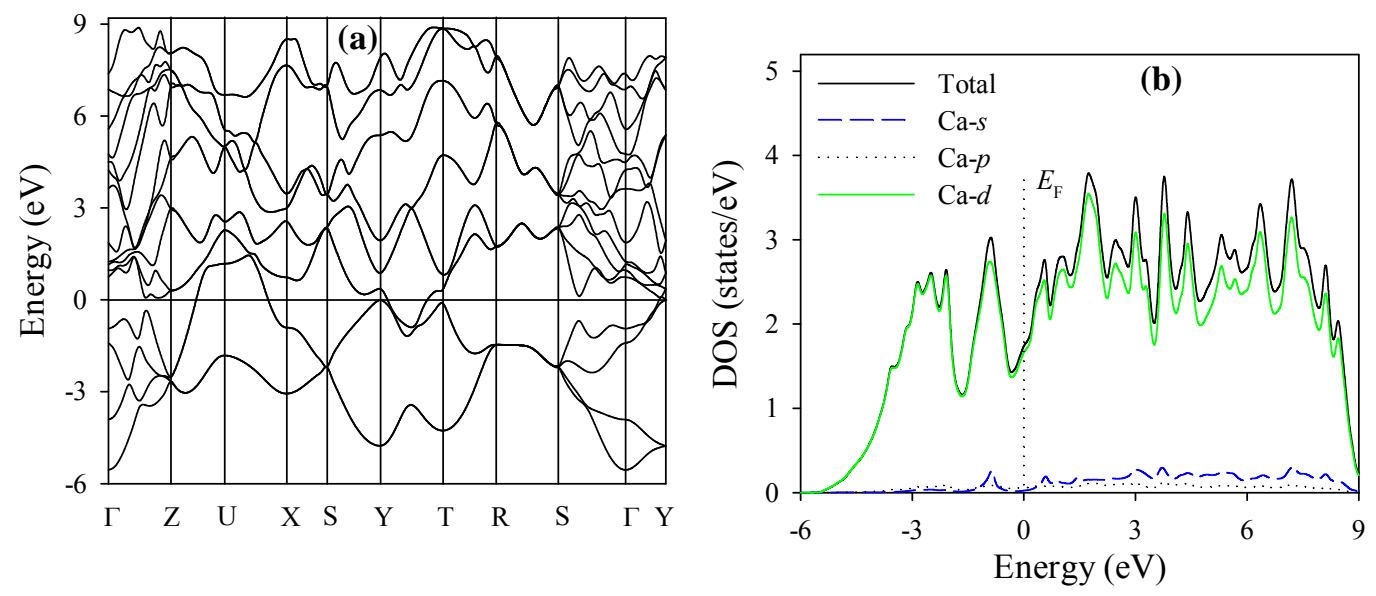

Figure 2. (a) Band structure, (b) (color online) total and projected DOS to $s, p$, and $d$ orbitals of $\mathrm{Ca}-\mathrm{VI}$ at $172 \mathrm{GPa}$.

\subsection{Phonon spectrum, electron-phonon coupling and superconductivity}

To investigate the occurrence of superconductivity, the phonon linewidths, EPC parameter have been calculated using perturbative linear response method within the density-functional approximation [30]. The phonon dispersion curves, and phonon density of states (PHDOS) as a function of frequency of the Pnma structure were evaluated at 158,161, 165, 172 and 180 GPa (Figure shown only for 161, $172 \mathrm{GPa}$ ). The Pnma structure is found to be stable in the entire pressure range of 158-180 $\mathrm{GPa}$ as indicated by the lack of imaginary frequencies. The two PHDOS show that higher frequency modes exist for $172 \mathrm{GPa}$ than $161 \mathrm{GPa}$. The PHDOS strength is distributed over frequency, having peaks for mid- and also high-range frequency phonons.
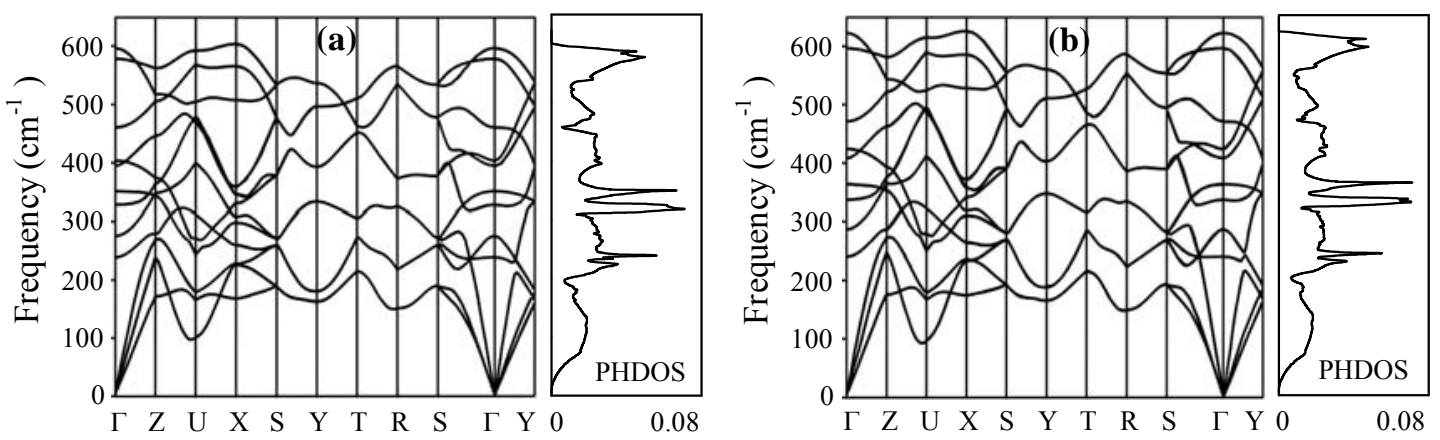

Figure 3. Phonon dispersion curves (left) and phonon DOS (right) at (a) 161 and (b) 172 GPa.

At $158 \mathrm{GPa}$, the calculated coupling constant $\lambda$ and phonon frequency logarithmic average $\omega_{\text {log }}$ for the Pnma phase are 0.896 and $383 \mathrm{~K}$, respectively. The corresponding values of the 
parameters at $161,165,172$, and $180 \mathrm{GPa}$ are: $0.903,408 \mathrm{~K} ; 0.915,405 \mathrm{~K} ; 0.934,396 \mathrm{~K}$ and $0887,411 \mathrm{~K}$, respectively. The superconducting critical temperature $T_{c}$ can be estimated from the Allen-Dynes modified McMillan equation [31]:

$$
k_{\mathrm{B}} T_{\mathrm{c}}=\frac{\hbar \omega_{\log }}{1.2} \exp \left[-\frac{1.04(1+\lambda)}{\lambda-\mu^{*}(1+0.62 \lambda)}\right]
$$

Using an empirical value 0.1 for the Coulomb pseudopotential $\mu^{*}$, the estimated $T_{\mathrm{c}}$ at 172 GPa is $24.7 \mathrm{~K}$ for the Ca-VI Pnma phase. We show a plot of calculated $T_{\mathrm{c}}$ versus $P$ in figure 4. The theoretically predicted maximum value occurs at $172 \mathrm{GPa}$ and is close to the experimentally observed $T_{\mathrm{c}}$ of $25 \mathrm{~K}$ at $161 \mathrm{GPa}$ [10].

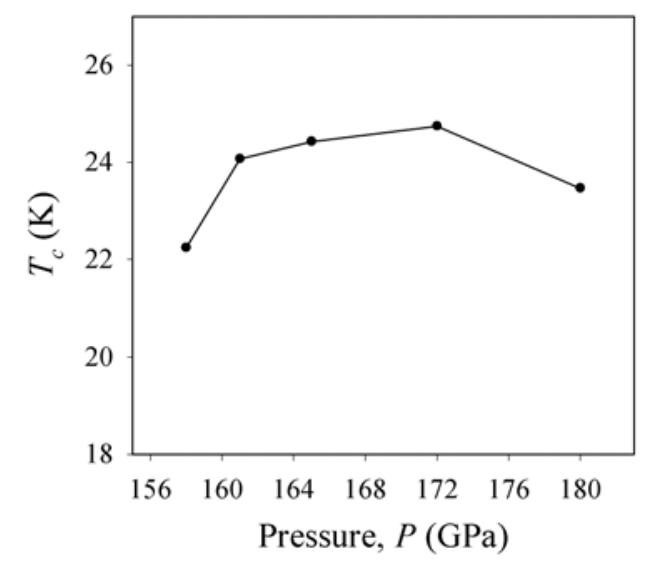

Figure 4. $T_{\mathrm{c}}$ versus pressure of Ca-VI (Pnma).

In fact there is some uncertainty in the estimation of $T_{\mathrm{c}}$ due to the lack of knowledge of the value of $\mu^{*}$ and its pressure dependence. Since the pressure dependence of $\mu^{*}$ is shown to be negligible [32] the only uncertainty is in the value of $\mu^{*}$ itself. Usually it is taken as 0.1- 0.13. Our calculated values may be slightly overestimated because of the Coulomb pseudopotential (0.1) but the trend of the resulting $T_{\mathrm{c}}$ passes near the experimental value at $161 \mathrm{GPa}$. The observed high $T_{\mathrm{c}}$ may be attributed to a combination of rather large $\lambda$ and that almost all phonon modes contribute to the EPC processes leading to a large $\omega_{\log }$.

The high-pressure electronic properties of $\mathrm{Ca}$ (see section $\mathrm{C}$ ) can be discussed in the light of pressure-induced $4 s$ - $3 d$ electronic transfer $[1,17,33]$. At high pressure, the $d$-bands near the Fermi surface shift downward to points where valence electrons in Ca transfer from $s$ bands to $d$-bands. The $d$-band occupation number ( $n_{\mathrm{d}}$ of electrons) determins the crystal stability at large compression. This is considered to be an indication of the strength of electron-phonon interaction leading to superconductivity. In the case of $\mathrm{Ca}-\mathrm{VI}, T_{\mathrm{c}}$ increases with pressure (with a maximum at $\sim 172 \mathrm{GPa}$ ) and then it decreases. Thus the $s-d$ electronic transfer at $172 \mathrm{GPa}$ may be thought to be completed [34].

\section{Conclusion}

The phase Ca-VI is in the Pnma phase which is seen to be mechanically and dynamically stable in the entire pressure range of 158-180 GPa. The calculated Zener's anisotropy index shows that $\mathrm{Ca}-\mathrm{VI}$ approaches towards isotropy as pressure increases. Further from an analysis of the elastic parameters of polycrystalline aggregates of $\mathrm{Ca}-\mathrm{VI}$, the material is found to behave as a hard metallic like system for the pressure range considered here. 
The superconducting critical temperatures $T_{\mathrm{c}}$ have been calculated at five pressures: 158, $161,165,172$ and $180 \mathrm{GPa}$. The theoretically predicted maximum value occurs at $172 \mathrm{GPa}$ and is close to the experimentally available $T_{\mathrm{c}}$ value of $25 \mathrm{~K}$ at $161 \mathrm{GPa}$. As pressure increases, the $d$-bands near the Fermi surface is found to shift downward to points where valence electrons in Ca transfer from $s$-bands to $d$-bands. Such transfer of charges at $\sim 172$ GPa may be thought to be completed, where the calculated $T_{\mathrm{c}}(24.7 \mathrm{~K})$ is found to be maximum.

\section{Acknowledgments}

The authors acknowledge the help received from Rajshahi University and Bangladesh University Grants Commission.

\section{References}

[1] Skriver H L 1982 Phys. Rev. Lett. 491768

[2] Nakamoto Y, Sakata M, Shimizu K, Fujihisa H, Matsuoka T, Ohishi Y, and Kikegawa T 2010 Phys. Rev. B 81 140106(R)

[3] Moodenbaugh A R and Wittig J 1973 J. Low. Temp. Phys. 10203

[4] Probst C and Wittig J 1977 Phys. Rev. Lett. 391161

[5] I'lina M A, Itskevich E S, and Dizhui E M 1972 JETP 341263

[6] Dunn K J and Bundy F P 1982 Phys. Rev. B 25194

[7] Gandel'man G M and Fedorov M A 1971 JETP Lett. 13127

[8] Dunn K J and Bundy F P 1981 Phys. Rev. B 241643

[9] Okada S, Shimizu K, Kobayashi T C, Amaya K, and Endo S 1996 J. Phys. Soc. Jpn 65 1924

[10] Yabuuchi T, Matsuoka T, Nakamoto Y, Shimizu K 2006 J. Phys. Soc. Jpn 75083703

[11] Lei S, Papaconstantopoulos D A, and Mehl M J 2007 Phys. Rev. B 75024512

[12] Arapan S, Mao H K, and Ahuja R 2008 Proc. Natl. Acad. Sci. U.S.A. 10520627

[13] Yao Y, Tse J S, Song Z, Klug D D, Sun J, and Le Page Y 2008 Phys. Rev. B 78 054506

[14] Ishikawa T, Nagara H, Suzuki N, Tsuchiya T, and Tsuchiya J 2010 Phys. Rev. B 81 092104

[15] Gao G, Xie Y, Cui T, Ma Y, Zhang L and Zou G 2008 Solid State Commun. 146181

[16] Yin Z P, Gygi F, and Pickett W E 2009 Phys. Rev. B 80184515

[17] Ahuja R, Eriksson O, Wills J M and Johansson B 1995 Phys. Rev. Lett. 753473.

[18] Clark S J, Segall M D, Pickard C J, P. J. Hasnip, Probert M I J, Refson K, and Payne M C 2005 Zeitschrift für Kristallographie 220567

[19] Perdew J P, Ruzsinszky A, Csonka G I, Vydrov O A, Scuseria G E, Constantin L A, Zhou X, and Burke K 2008 Phys. Rev. Lett. 100136406

[20] Giannozzi P, Baroni S, Bonini N, Calandra M, Car R, Cavazzoni C, Ceresoli, Chiarotti D L, Cococcioni M, Dabo I, Dal Corso A, de Gironcoli S, Fabris S, Fratesi G, Gebauer R, Gerstmann U, Gougoussis C, Kokalj A, Lazzeri M, Martin-Samos L, Marzari N, Mauri F, Mazzarello R, Paolini S, Pasquarello A, Paulatto L, Sbraccia C, Scandolo S, Sclauzero G, Seitsonen A P, Smogunov A, Umari P, and Wentzcovitch R M $2009 \mathrm{~J}$. Phys.: Condens.Matter 21395502

[21] D. Vanderbilt 1990 Phys. Rev. B 417892

[22] Born M and Huang K 1956 Dynamical Theory of Crystal Lattices (Clarendon, Oxford).

[23] Zener C 1948 Elasticity and Anelasticity of Metals (Univesity of Chicago, Chicago)

[24] Voigt W 1928 Lehrbuch der Kristallphysik (Teubner,Leipzig) 716 [in German].

[25] Reuss A 1929 Z. Angew. Math. Mech. 949

[26] Hill R 1952 Proc. Phys. Soc., London, Sect. A 65349

[27] Hossain M A, Islam A K M A, and Islam F N 2009 J. Sci. Res. 1 (2) 182

[28] Pugh S F 1953 Phil. Mag. 45833 
[29] Haines J, Leger J, and Bocquillo G 2001 Ann. Rev. Mater. Res. 31

[30] Baroni S, de Gironcoli S, Dal Corso A, and Giannozzi P 2001 Rev. Mod. Phys. 73 515; http://www.pwscf.org

[31] Allen P B and Dynes R C 1975 Phys. Rev. B 12905

[32] Chen X J, Zhang H and Habermeier H -U 2002 Phys. Rev. B 65144514

[33] McMahan A K 194 Phys. Rev. B 295982

[34] Nelmes R J, Allan D R, McMahon M I, and Belmonte S A 1999 Phys. Rev. Lett. 83 4081 